\title{
THE RELATIONSHIP BETWEEN POLAR AND AC OPERATORS
}

\author{
JULIE WILSON \\ Department of Mathematics, Glasgow Caledonian University, Cowcaddens Road, Glasgow G4 OBA, \\ Scotland \\ (Received 7 January, 1998)
}

\begin{abstract}
This paper gives examples to show that a polar operator is not necessarily $\mathrm{AC}$ and an $\mathrm{AC}$ operator is not necessarily polar.

1. Introduction. Well-bounded operators are the building blocks of polar and AC operators. First introduced by Smart in 1960, well-bounded operators were originally studied by Smart and Ringrose [6], [7], [8]. These operators admit a spectral decomposition which is, in some sense, analogous to that for self-adjoint operators on Hilbert space. The spectral decomposition is simplified when we consider wellbounded operators of type (B).

By their definition, well-bounded operators have real spectra. In order to extend the concept of well-boundedness to operators with complex spectra, we consider trigonometrically well-bounded, polar and AC operators. The relevant facts about well-bounded, polar and AC operators are outlined in the next section. For a detailed account of the theory well-bounded operators see [4].
\end{abstract}

2. Background and notation. Throughout the following $X$ will denote a complex Banach space with dual space $X^{*}$ and $\mathcal{B}(X)$ will denote the algebra of all bounded linear operators mapping $X$ into itself. Given a compact interval $J=[a, b]$ of the real line, let $B V(J)$ denote the Banach algebra of complex-valued functions of bounded variation on $J$ with norm

$$
\|f\|_{B V(J)}=|f(b)|+\underset{J}{\operatorname{var} f}
$$

where $\underset{J}{\operatorname{var} f}$ represents the total variation of $f$ on $J$. Similarly, using $\mathbf{T}$ to represent the unit circle, let $B V(\mathbf{T})$ denote the Banach algebra of complex-valued functions of bounded variation on $\mathbf{T}$ with norm

$$
\|f\|_{B V(\mathbf{T})}=|f(1)|+\underset{\mathbf{T}}{\operatorname{var}} f,
$$

where $\underset{\mathbf{T}}{\operatorname{var} f} f$ is the total variation of $f$ on $\mathbf{T}$. Furthermore, the notation $A C(J)$ (respectively $A C(\mathbf{T})$ ) will denote the closed subalgebra of $B V(J)$ (respectively $B V(\mathbf{T})$ ) consisting of the absolutely continuous functions on $J$ (respectively $\mathbf{T}$ ).

Definition 2.1. An operator $T \in \mathcal{B}(X)$ is said to be well-bounded if there exists a constant $K$ and a compact interval $J \subseteq \mathbf{R}$ such that

$$
\|p(T)\| \leq K\|p\|_{B V(J)},
$$

for all polynomials $p$. 
Note that, in this case, the spectrum of $T$ must be a subset of $J$.

Definition 2.2. Let $J$ be a compact interval of the real line. An $A C(J)$-functional calculus (respectively an $A C(\mathbf{T})$-functional calculus) for an operator $T \in \mathcal{B}(X)$ is a norm-continuous algebra-homomorphism $\gamma$ of $A C(J)$ into $\mathcal{B}(X)$ (resp. $A C(\mathbf{T})$ into $\mathcal{B}(X)$ ) which sends the identity map $v(t)=t$ to $T$ and the function identically 1 to $I$, the identity operator of $\mathcal{B}(X)$. In addition, $\gamma$ is said to be weakly compact if, for each $x \in X, \gamma(\cdot) x$ is a weakly compact linear mapping of the domain of $\gamma$ into $X$.

Since the polynomials are dense in the set of absolutely continuous functions [7, Lemma 10], we can say that an operator $T$ is well-bounded if there exists a compact interval $J$ for which $T$ has an $A C(J)$-functional calculus.

Definition 2.3. An operator $T$ is said to be well-bounded of type (B) if, for some compact interval $J, T$ has a weakly compact $A C(J)$-functional calculus. (Note that if $X$ is a reflexive space then every well-bounded operator on $X$ is automatically of type (B). See [5, p. 68].)

Definition 2.4. A spectral family in $X$ is a projection-valued function $E(\cdot): \mathbf{R} \rightarrow \mathcal{B}(X)$ satisfying the following conditions:

(i) $\sup \{\|E(\lambda)\|: \lambda \in \mathbf{R}\}<\infty$;

(ii) $E(\lambda) E(\mu)=E(\mu) E(\lambda)=E(\min \{\lambda, \mu\})(\lambda, \mu, \in \mathbf{R})$;

(iii) $E(\cdot)$ is strongly right continuous;

(iv) $E(\cdot)$ has a strong left-hand limit at each point of $\mathbf{R}$;

(v) $E(\lambda) \rightarrow 0$ (respectively $E(\lambda) \rightarrow I$ ) in the strong operator topology of $\mathcal{B}(X)$ as $\lambda \rightarrow-\infty$ (respectively $\lambda \rightarrow+\infty$ ).

Note. If $E(\lambda)=0$ for all $\lambda<a$, and $E(\lambda)=I$ for all $\lambda \geq b$, then $E(\cdot)$ is said to be concentrated on $[a, b]$.

If $E(\cdot)$ is a spectral family in $X$ concentrated on $J=[a, b]$ and $f \in B V(J)$, then

$$
\int_{J}^{\oplus} f(\lambda) d E(\lambda) \equiv f(a) E(a)+\int_{a}^{b} f(\lambda) d E(\lambda)
$$

exists as the strong limit of the Riemann-Stieltjes sums

$$
\mathcal{S}(f, u)=f(a) E(a)+\sum_{j=1}^{n} f\left(\lambda_{j}\right)\left\{E\left(\lambda_{j}\right)-E\left(\lambda_{j-1}\right)\right\},
$$

where $u=\left(\lambda_{0}, \lambda_{1}, \ldots, \lambda_{n}\right)$ is a partition of $J$. Rearranging the above in the style of integration by parts gives

$$
\mathcal{S}(f, u)=f(b) E(b)-\sum_{j=1}^{n}\left\{f\left(\lambda_{j}\right)-f\left(\lambda_{j-1}\right)\right\} E\left(\lambda_{j-1}\right) .
$$

The following results concerning well-bounded operators maybe found in $[4$, Part V]. 
Proposition 2.5. The mapping

$$
f \rightarrow \int_{J}^{\oplus} f d E
$$

is an identity-preserving algebra homomorphism of $B V(J)$ into $\mathcal{B}(X)$ satisfying

$$
\left\|\int_{J}^{\oplus} f d E\right\| \leq\|f\|_{B V(J)} \sup \{\|E(\lambda)\|: \lambda \in \mathbf{R}\}
$$

for every $f \in B V(J)$.

Proposition 2.6. Let $T \in \mathcal{B}(X)$. Then $T$ is well-bounded of type $(B)$ if and only if there exists a spectral family $E(\cdot)$ in $X$ such that

(i) $E(\cdot)$ is concentrated on a compact interval $[a, b]$, and

(ii) $T=\int_{[a, b]}^{\oplus} \lambda d E(\lambda)$.

In this case $E(\cdot)$ is uniquely determined and is called the spectral family of $T$.

Proposition 2.7. Let $T \in \mathcal{B}(X)$ be well-bounded of type $(B)$ and let $E(\cdot)$ be its spectral family. Then an operator $S$ commutes with $T$ if and only if $S$ commutes with $E(\lambda)$, for all $\lambda \in \mathbf{R}$.

Proposition 2.8. Let $T \in \mathcal{B}(X)$ be well-bounded of type $(B)$ and let $E(\cdot)$ be its spectral family. Then for each $\lambda \in \mathbf{R},\left\{E(\lambda)-E\left(\lambda^{-}\right)\right\}$is a projection operator and

$$
\left\{E(\lambda)-E\left(\lambda^{-}\right)\right\} X=\{x \in X: T x=\lambda x\}
$$

where $E\left(\lambda^{-}\right)$denotes the strong limit of $E(s)$ as $s \rightarrow \lambda^{-}$.

Trigonometrically well-bounded, polar and AC operators all arise from wellbounded operators. Their definitions are given below.

Definition 2.9. An operator $T \in \mathcal{B}(X)$ is said to be trigonometrically wellbounded if there exists a well-bounded operator $A$ of type $(B)$ on $X$ such that $T=e^{i A}$.

Proposition 2.10. If $T$ is a trigonometrically well-bounded operator on the Banach space $X$, then there is a unique well-bounded operator A of type (B) on X such that $T=e^{i A}, \sigma(A) \subseteq[0,2 \pi]$, and such that $\sigma_{p}(A)$, the point spectrum of $A$, does not contain $2 \pi$.

Definition 2.11. The unique operator $A$ in Proposition 2.10 is called the $\operatorname{argu-}$ ment of $T$ and is denoted by $\arg T$. For more on trigonometrically well-bounded operators see [3].

Definition 2.12. An operator $T \in \mathcal{B}(X)$ is said to be a polar operator if there exist commuting type $(B)$ well-bounded operators $R$ and $A$ on $X$ such that $T=R e^{i A}$. The following results about polar operators will be required in Section 3 . 
TheOrem 2.13. See [1, Theorem 1]. Let $T \in \mathcal{B}(X)$ be polar. Then $T$ has a decomposition $T=R e^{i A}$ such that

(i) $R$ and $A$ are commuting well-bounded operators of type (B);

(ii) $\sigma(R) \geq 0$;

(iii) $F(0) e^{i A}=F(0)$, where $F(\cdot)$ is the spectral family of $R$;

(iv) $\sigma(A) \subseteq[0,2 \pi], 2 \pi \notin \sigma_{p}(A)$.

This decomposition is unique and is called the canonical decomposition of $T$.

THeOREM 2.14. See [1, Theorem 3.18(i)]. Let T be a polar operator with canonical decomposition $T=R e^{i A}$. Then the commutants of $T, R$ and $A$ satisfy the equality $\{T\}^{\prime}=\{R\}^{\prime} \cap\{A\}^{\prime}$.

Polar operators are discussed further in [1] and [9]. The final definition required is that of an $\mathrm{AC}$ operator.

Definition 2.15. An operator $T \in \mathcal{B}(X)$ is said to be an $A C$ operator if there exist commuting well-bounded operators $C$ and $D$ such that $T=C+i D$. AC operators are studied in [2], from which the following result is taken.

Theorem 2.16. See [2, Lemma 4]. Let $C$ and $D$ be commuting well-bounded operators of type $(B)$ on $X$ and let $S \in \mathcal{B}(X)$ commute with $C+i D$. Then $S$ commutes with $C$ and $D$.

It has been shown [3, Theorem 3.4] that an operator $T$ is trigonometrically wellbounded if and only if there exist commuting well-bounded operators $A$ and $B$ of type (B) such that

$$
T=A+i B
$$

and

$$
A^{2}+B^{2}=I
$$

With this in mind, it seems natural to pose the following questions.

(1) If $T$ is polar, do there exist commuting well-bounded operators $A$ and $B$ (of type (B)) such that (1) holds?

(2) If $T=A+i B$ with $A$ and $B$ commuting well-bounded operators of type (B), does it follow that $T$ is polar?

We shall now give examples to show that the answer to both these questions is negative. We shall use the following definitions and results from [4].

3. Examples. Let $a \in l^{2}$ and, for $n \in N$, let $P_{n}: l^{2} \rightarrow l^{2}$ be defined by

$$
P_{n} a=\left\langle a, y_{n}\right\rangle x_{n},
$$

where 


$$
\begin{aligned}
x_{2 n-1} & =e_{2 n-1}+\sum_{i=n}^{\infty} \alpha_{i-n+1} e_{2 i}, \\
x_{2 n} & =e_{2 n}, \\
y_{2 n-1} & =e_{2 n-1}, \\
y_{2 n} & =\sum_{i=1}^{n}\left(-\alpha_{n-i+1}\right) e_{2 i-1}+e_{2 n}(n \in \mathbf{N}), \\
\alpha_{1} & =0, \alpha_{n}=\frac{1}{n \log n},(n=2,3, \ldots),
\end{aligned}
$$

and $\varepsilon_{n}$ is the element of $l^{2}$ with 1 in its $n$th position and 0 elsewhere. Then each $P_{n}$ is a projection, $P_{n} P_{m}=0$ whenever $n \neq m$, and $I=\sum_{n=1}^{\infty} P_{n}$, the series converging in the strong operator topology of $\mathcal{B}\left(l^{2}\right)$.

Proposition 3.1. See $([\mathbf{4}, 18.5])$. Let $\left\{\lambda_{n}\right\}$ be a monotonic bounded sequence in $\mathbf{R}$ and, for each $n \in \mathbf{N}$, let $P_{n}$ be as above. Then the series $\sum_{n=1}^{\infty} \lambda_{n} P_{n}$ converges strongly in $\mathcal{B}\left(l^{2}\right)$ and its sum is a well-bounded operator.

Note that in the proof of 18.4 in [4] it is shown that

$$
\left\|\sum_{j=1}^{n} P_{2 j}\right\| \rightarrow \infty \text { as } n \rightarrow \infty .
$$

We shall use this result in the example below.

Example. Let $X=l^{2}, P_{n}$ be defined as above, and define sequences $\left\{\lambda_{n}\right\}$ and $\left\{\mu_{n}\right\}$ by

$$
\lambda_{n}=\frac{n+1}{n}
$$

and

$$
\mu_{2 n-1}=\mu_{2 n}=\cos ^{-1}\left(\frac{4 n^{2}-1 / 2}{4 n^{2}+2 n}\right),
$$

for all $n \in \mathbf{N}$. In addition, define

$$
C=\sum_{n=1}^{\infty} \lambda_{n} P_{n} \text { and } D=\sum_{n=1}^{\infty} \mu_{n} P_{n}
$$

By Proposition 3.1, each series converges strongly in $\mathcal{B}\left(l^{2}\right)$ and $C$ and $D$ are wellbounded operators (of type (B)). Furthermore, since $C$ and $D$ commute, it follows that $C e^{i D}$ is polar. 
Now suppose that $C \cos D$ is well-bounded with spectral family $E(\cdot)$. Then

$$
\begin{aligned}
(C \cos D) P_{n} & =\left(\lambda_{n} \cos \mu_{n}\right) P_{n} \\
& = \begin{cases}\left(\frac{n^{2}-1 / 2}{n^{2}}\right) P_{n} & \text { when } n \text { is even } \\
\left(\frac{n^{2}+2 n+1 / 2}{n^{2}+2 n}\right) P_{n} & \text { when } n \text { is odd } .\end{cases}
\end{aligned}
$$

Fix $x \in l^{2}$ and suppose that $n$ is even. Observe that

$$
P_{n} x \in\left\{x \in l^{2}:(C \cos D) x=\left(\frac{n^{2}-1 / 2}{n^{2}}\right) x\right\} .
$$

Furthermore, if

$$
(C \cos D) x=\frac{n^{2}-1 / 2}{n^{2}} x,
$$

then

$$
P_{m} x=\frac{n^{2}}{n^{2}-1 / 2}(C \cos D) P_{m} x
$$

and it follows that $P_{m} x=0$ if $m \neq n$. Thus

$$
\left\{x \in l^{2}:(C \cos D) x=\left(\frac{n^{2}-1 / 2}{n^{2}}\right) x\right\} \subseteq P_{n} X
$$

Combining statements (4) and (5), we see that

$$
P_{n} X=\left\{x \in l^{2}:(C \cos D) x=\left(\frac{n^{2}-1 / 2}{n^{2}}\right) x\right\} .
$$

Also, by Proposition 2.8,

$$
P_{n} X=\left\{E\left(\frac{n^{2}-1 / 2}{n^{2}}\right)-E\left(\left(\frac{n^{2}-1 / 2}{n^{2}}\right)\right)\right\} X .
$$

Since

$$
\frac{n^{2}-1 / 2}{n^{2}}<1
$$

for all $n \in \mathbf{N}$, it follows that $E(1) P_{n}=P_{n}$ whenever $n$ is even.

When $n$ is odd, an argument similar to that above shows that

$$
P_{n} X=\left\{x \in l^{2}: C \cos D x=\left(\frac{n^{2}+2 n+1 / 2}{n^{2}+2 n}\right) x\right\} .
$$

As

$$
\frac{n^{2}+2 n+1 / 2}{n^{2}+2 n}>1
$$

for all $n \in \mathbf{N}$, it follows that $E(1) P_{n}=0$ for $n$ odd. 
Combining even and odd cases, we see that

$$
E(1)=\sum_{n=1}^{\infty} E(1) P_{n}=\sum_{n=1}^{\infty} P_{2 n},
$$

with the series converging in the strong operator topology of $\mathcal{B}\left(l^{2}\right)$. Since $E(1)$ is bounded, the partial sums of the series $\sum_{n=1}^{\infty} P_{2 n}$ must be bounded in norm, giving a contradiction to (3). Hence $C \cos D$ is not well-bounded.

Now suppose that $T=C e^{i D}=A+i B$, where $A$ and $B$ are commuting wellbounded operators. Since $C$ and $D$ commute with $T$ it follows that $C \cos D$ commutes with $T$ and, by Theorem 2.16, $C \cos D$ commutes with $A$ and $B$.

Next fix $n \in \mathbf{N}$ and suppose that $y \in P_{n} X=\left\{x \in X: C \cos D x=\lambda_{n} \cos \mu_{n} x\right\}$. Then

$$
A y=\left(\lambda_{n} \cos \mu_{n}\right)^{-1} A(C \cos D) y=\left(\lambda_{n} \cos \mu_{n}\right)^{-1}(C \cos D) A y
$$

and hence $A y \in P_{n} X$. A similar argument shows that $P_{n} X$ is invariant under $B$. It readily follows that $A$ and $B$ commute with $P_{n}$.

Now, since each $P_{n} X$ is one-dimensional (the $P_{n} X$ are the eigenspaces of $C \cos D$ corresponding to the distinct eigenvalues $\lambda_{n} \cos \mu_{n}$ ), there exist $\alpha_{n}$ and $\beta_{n} \in \mathbf{R}$ such that

$$
A \mid P_{n} X=\alpha_{n} \text { and } B \mid P_{n} X=\beta_{n} .
$$

Thus, on $P_{n} X$, we have

$$
T=C e^{i D}=\lambda_{n} \cos \mu_{n}+i \lambda_{n} \sin \mu_{n}
$$

and also

$$
T=A+i B=\alpha_{n}+i \beta_{n}
$$

Equating real and imaginary parts gives $\alpha_{n}=\lambda_{n} \cos \mu_{n}$ and $\beta_{n}=\lambda_{n} \sin \mu_{n}$. Thus $A=C \cos D$ on each $P_{n} X$ and it follows that $A=C \cos D$. This contradicts the fact that $A$ is well-bounded. Hence $T$ cannot be AC.

An example of an AC operator which is not polar now follows. define

Example. Let $X=l^{2}$ and, for $n \in \mathbf{N}$, let $P_{n}$ be as in the example above. Now

$$
C=\sum_{n=1}^{\infty} \lambda_{n} P_{n} \text { and } D=\sum_{n=1}^{\infty} \mu_{n} P_{n},
$$

where, for $n \in \mathbf{N}$,

$$
\lambda_{n}=\sqrt{\frac{n+1}{n}}
$$

and

$$
\mu_{2 n-1}=\mu_{2 n}=\sqrt{\frac{(2 n-1)-(4 n-2)^{-1}}{2 n}} .
$$


By Proposition 3.1, $C$ and $D$ are well-bounded operators (of type (B)). Moreover, as $C$ and $D$ commute, $T=C+i D$ is AC.

Now suppose that $T$ is polar with canonical form $R e^{i A}$. By Theorems 2.14 and 2.16, $R$ and $A$ commute with $C$ and $D$. It is readily checked that, for each $n \in \mathbf{N}, P_{n} X=\left\{x \in X: C x=\lambda_{n} x\right\}, P_{n} X$ is invariant under both $R$ and $A$, and $P_{n}$ commutes with $R$ and $A$.

Now, given $n \in \mathbf{N}$, there exist $r_{n}$ and $\theta_{n} \in \mathbf{R}$ such that

$$
R \mid P_{n} X=r_{n} \text { and } A \mid P_{n} X=\theta_{n} .
$$

Furthermore, on $P_{n} X$,

$$
T=R e^{i A}=r_{n} e^{i \theta_{n}}
$$

and

$$
T=C+i D=\lambda_{n}+i \mu_{n}
$$

Hence $r_{n} e^{i \theta_{n}}=\lambda_{n}+i \mu_{n}$ and $r_{n}=\left(\lambda_{n}^{2}+\mu_{n}^{2}\right)^{1 / 2}$, for all $n \in \mathbf{N}$. Observe that

$$
r_{n}= \begin{cases}\left(2-\frac{1}{2 n(n-1)}\right)^{1 / 2} & \text { for } n \text { even } \\ \left(\frac{2 n^{2}+2 n+1 / 2}{n^{2}+n}\right)^{1 / 2} & \text { for } n \text { odd }\end{cases}
$$

so that $r_{n}<\sqrt{2}$ if $n$ is even and $r_{n}>\sqrt{2}$ if $n$ is odd. Notice also that

$$
P_{n} X=\left\{x \in X: R x=r_{n} x\right\} .
$$

If $E(\cdot)$ is the spectral family of $R$ then $E(\sqrt{2})$ is a bounded projection on $X$ and, by Proposition 2.8 and 2.4(ii),

$$
\begin{aligned}
E(\sqrt{2}) P_{n} X & =E(\sqrt{2})\left\{E\left(r_{n}\right)-E\left(r_{n}^{-}\right)\right\} X \\
& = \begin{cases}\left\{E\left(r_{n}\right)-E\left(r_{n}^{-}\right)\right\} X & \text { for } n \text { even, } \\
0 & \text { for } n \text { odd. }\end{cases}
\end{aligned}
$$

It follows that

$$
E(\sqrt{2}) P_{n}= \begin{cases}P_{n} & \text { for } n \text { even } \\ 0 & \text { for } n \text { odd }\end{cases}
$$

This gives

$$
E(\sqrt{2})=\sum_{n=1}^{\infty} E(\sqrt{2}) P_{n}=\sum_{n=1}^{\infty} P_{2 n},
$$

where the series converges in the strong operator topology of $\mathcal{B}\left(l^{2}\right)$. As $E(\sqrt{2})$ is bounded, the partial sums of the series $\sum_{n=1}^{\infty} P_{2 n}$, must be bounded in norm. Again we have a contradiction to (3) so that $R$ cannot be well-bounded and $T$ is not polar. 


\section{REFERENCES}

1. H. Benzinger, E. Berkson and T. A. Gillespie, Spectral families of projections, semigroups, and differential operators, Trans. Amer. Math. Soc., 275 (1983), 431-475.

2. E. Berkson and T. A. Gillespie, Absolutely continuous functions of two variables and well-bounded operators, J. London Math. Soc., 30 (1984), 305-321.

3. E. Berkson and T. A. Gillespie, AC functions on the circle and spectral families. $J$. Operator Theory, 13 (1985), 33-47.

4. H. R. Dowson, Spectral theory of linear operators (Academic Press, 1978). 1957).

5. N. Dunford and J. T. Schwartz, Linear operators Part 1: General theory (Interscience,

6. J. R. Ringrose, On well-bounded operators, J. Austral. Math. Soc., 1 (1960), 334-343.

7. J. R. Ringrose, On well-bounded operators II, Proc. London Math. Soc. (3), 13 (1963), 613-638.

8. D. R. Smart, Conditionally convergent spectral expansions. J. Austral. Math. Soc., 1 (1960), 319-333.

9. J. Wilson, Polar and AC operators, the Hibert transform, and matrix-weighted shifts, $\mathrm{Ph}$. D. Thesis (University of Edinburgh, 1997). 